\title{
Pressurized Liquid Filling Method
}

National Cancer Institute

\section{Source}

National Cancer Institute. Pressurized Liquid Filling Method. NCI Thesaurus. Code

C112999.

A process that fills a dosage unit with a pressurized liquid, which is not performed under sterile conditions. 